\title{
Federated Sensor Network Prospects and Challenges
}

\section{Adnan Al-Anbuky*}

Sensor Network \& Smart Environment Research Lab, School of Engineering, Auckland University of Technology, New Zealand

The area of Wireless Sensor Network WSN is considered as one of the most important and fast growing fields, penetrating through almost every aspect of life. Modern enabling technologies like the Internet of Things IoT and Cloud Computing have opened up more doors for emphasizing the importance of the WSN field. Research and development of concepts related to sensor networks are driving towards taking the experiments from the laboratory into the field, with emphasis on various modes of communication including Opportunistic Communication and Machine-to-Machine communication. A wide range of testbeds has been established for remotely testing design ideas on various physical environments [1-5]. These testbeds have been mostly driven by network and communication groups and have created real test environments and stimulated research into related architectures and solutions. Virtual support for extending the capabilities of such environments has also been explored. This aims at enabling the mix and match among available resources of multiple testbeds in formulating a workable federation [6]. Areas like data and reality mining, cyber-physical systems [7] and others have started pushing towards the formulation of highly complex systems. These systems are centered round the Internet and sensor networks and are driving towards what has been referred to as the planet nervous system [8]. While elements of the concept have started taking shape, there are significant operational and optimization challenges. The synergy among the various acting subsystems, the redundancies of multiple solutions on the same physical space, and the physical and radio pollution impacting the living spaces are just to name few.

Early concepts relevant to the internet of things go back as far as the late 90 's. The challenge at the time was to enable embedded controllers to communicate over the TCP IP Protocol. The Dallas Tini Internet interface is a typical example [9]. Today's microcontroller industries are racing in securing the $6 \mathrm{LoWPAN}$ protocol as an Internet enabler within their wireless microcontroller products. This has opened the door for a range of options and has stimulated number of areas of research. The most obvious is in relation to smart devices. Association with a sensor network has been made through the low energy protocols like the 6LoWPAN or CoAP [10]. The challenge here is the tradeoff between the access to the Internet and efficient communication of a group of close-by sensors. This in effect encourages research on optimizing the way the sensor nodes connect to the Internet (i.e. as individuals or groups). The area may well be relevant to that of cyber-physical systems [7] as it relates to energy and communication performance. Open resources available at this stage require significant technical and specialized efforts before one can deal with their own concept. This makes it somehow difficult or prohibitive for a range of researchers. On the other hand progress here does open the doors for researchers to formulate perceptions and model concepts and solutions at simulation level. Recent literature started looking at incorporating semantics web in addressing the type of sensors or sensing [10]. Other aspects relevant to the analysis of the Quality of Service QoS and looking into the impact of enabling the various services through the IoT include throughput, energy used lifetime and Packet delivery [11]. The study revealed that energy used for the IoT is of the most critical differentiator among the various services. This is an area that will see more involved research into the optimization of communication system organization.

Another area of interest that benefits both scientific analysis as much as the sensor network system development community is that of mixed mode modelling involving physical system, simulation and emulation [12]. This is an area where lab work could get close to a real life system. Good progress is taking place here in facilitating an environment for testing concepts. The IBM MoteRunner environment is one of the examples here. Mixed mode modelling could be realized for a given wireless sensor network target [13]. On the other hand literature has also looked into pure virtualization using cloud computing. The Sensor-Cloud concept has been suggested by Alamri et al. [13] for interfacing the sensor network to the various related services as enabled by the Internet. This is another dimension to the development of sensor network systems and may even be extended to cover federated sensor networks. Services that relate to hard-real-time or machine-tomachine communication may be disadvantaged here due the latency of mixed mode communication systems as well as the virtualization overhead. The research challenge here is that of integrating the sensorcloud virtualization with the real time in-network data fusion. The organization may resolve the hard-real-time requirement and offer a more comprehensive range of services.

In conclusion the field of wireless sensor network has gone a long way toward highly complex system organization. This has benefited from range of sensing, ICT and communication technologies. A lot of effort has been put in place for facilitating physical environments that allow researchers to test their ideas. The rapid growth in this area has placed significant challenges on the maturity of these physical sensor network testbeds. It remains important that these testbeds are associated with a significant sector of services like weather systems, horticultural systems, construction industries, and others. The added advantage here is the involvement of scientists relevant to the end usage and hence presenting more realistic and dependable cases. Integration of IoT and Cloud computing into the overall sensor network system has opened up significant challenges for researchers to resolve. While the majority of sensor network research at this stage is handled by network and communication researchers, adding the focus of specialized services should encourage better optimization and more realistic solutions.

\section{References}

1. Ertin E. Arora A, Ramnath R, Nesterenko M, Naik V, et al (2006) Kansei: A Testbed for sensing at scale Information Processing in Sensor Networks. The Fifth International Conference.

2. http://wisebed.eu/site/

3. Werner-Allen G, Swieskowski P, Welsh M MoteLab: A Wireless Sensor Network Testbed.

4. http://www.crew-project.eu/twist

5. http://www.crew-project.eu/wilabt

*Corresponding author: Adnan Al-Anbuky, Sensor Network \& Smart Environment Research Lab, School of Engineering, Auckland University of Technology, New Zealand, E-mail: Adnan.anbuky@aut.ac.nz

Received July 19, 2013; Accepted July 22, 2013; Published July 24, 2013

Citation: Anbuky AA (2013) Federated Sensor Network Prospects and Challenges. J Telecommun Syst Manage 2: e108 doi:10.4172/2167-0919.1000e108

Copyright: (c) 2013 Anbuky AA. This is an open-access article distributed under the terms of the Creative Commons Attribution License, which permits unrestricted use, distribution, and reproduction in any medium, provided the original author and source are credited. 
Citation: Anbuky AA (2013) Federated Sensor Network Prospects and Challenges. J Telecommun Syst Manage 2: e108 doi:10.4172/2167$0919.1000 \mathrm{e} 108$

6. Coulson G, Porter B, Chatzigiannakis I, Fischer S,Pfisterer D, et al (2012) Flexible Experimentation in Wireless Sensor Networks. Commun ACM 55: 82 90.

7. Song Z, Sastry CR, Chen Y, Tas NC (2009) Optimal Observation for Cyberphysical Systems.

8. http://www.ibm.com/smarterplanet/us/en/overview/article/iot_video.html

9. http://www.futurlec.com/News/Dallas/InternetlC.html

10. Pfisterer D, Romer K, Bimschas D, Kleine O, Mietz R (2011) SPITFIRE: Towards a Semantic Web of Things IEEE Commun Magazine 40-48.
11. Nef MA, Kikiras PK (2012) Enabling QoS in the loT. Mt Blunc France 33-38.

12. Gluhak A, Krco S, Nati M, Pfisterer D, Mitton N, et al (2011) A Survey on facilities for experimental internet of things research IEEE Communication Magazine 49: 58-67.

13. Alamri A, Ansari WS, Hassan MM, Hossain SM, Alelaiwi AH (2013) A survey on Sensor-Cloud; Architecture Applications and Approaches. Int J Distri Sen Network. 